\title{
Stellenregister (Auswahl)
}

\section{Bibel}

\section{Altes Testament}

\section{Genesis}

1,20-2,19 65

1,31126

$2,796,139$

2,25-4,1 89

3143

3,1 142

3,1589

3,2463

6144

6,5144

$8,8-12 \quad 57$

$22,3 \quad 57$

2895

28,12 116

30,14126

30,14-16 89

37,336

39,14-15 7

49,8-12 191

$49,956,94,96,122,125$

49,9-10 138

49,11 112

49,14-15 57

Exodus

13,3058

19,458

$20,17 \quad 57$

34,2058

Levitikus

1165

11,1-38 66, 67, 76, 77

11,6155

11,1358

11,17-18 67, 74, 75, 87

11,1967

$12,8 \quad 57$

14,14-17 57

14,2257

22,2271

Numeri

12,1573

12,2273

21,6-8 142, 143

22,22-33 57

22,2363

$23,2268,69,140$

$24,868,141$

33,17141

Deuteronomium

5,1357

5,2157

1465
14,3-21 66, 67, 76, 77

14,7-8 155

14,16-17 18

14,17-18 67, 74, 75

32,1158

$33,1768,69$

33,20-22 56

Josua

5,13-15 63

Richter

14,5-6 56, 146

1. Buch Samuel

17,3656

25,42 57

2. Buch Samuel

1,2356

1. Buch der Könige

10,18-20 56

17,2196

2. Buch der Könige

4,24 57

1. Chronikbuch

24,15 58

Nehemia

10,2158

Tobit

12167

ljob

4,11a 70, 71

10,9109

12,7-9 86

$20,12-16^{\mathrm{Vg}} 170$

24,4 57

$39,5-9 \quad 57,59$

39,968

39,19-25 57

40,25-32 112, 114

Psalmen

2,2 114

7,356

$9,30^{\mathrm{LXX}} 95$

$16,9-10\left(15,9-10^{\mathrm{LXX}}\right) 107$

$17\left(16^{\mathrm{Vg}}\right) 191$

$17,34^{\text {LXX }} 73$

18,10-11 112

19,286

$22,14\left(21,14^{\mathrm{Vg}}\right) 146$

$22,22\left(21,22^{\mathrm{LXX}}\right) 68-69,140$

$23\left(22^{\mathrm{LXX}}\right) 108$

$24\left(23^{\mathrm{LXX}}\right) 96,101-102,105-108$

24,7-10 95, 96, 102, 105-109, 112, 114-119

$24,7-10\left(23,7-10^{\mathrm{LXX}}\right) 107$

$28,6^{\text {LXX }} 68,69$

$29,6\left(28,6^{\mathrm{LXX}}\right) 140,141$

$42,2\left(41,2^{\mathrm{LXX}}\right) 63,72,73,77,133,134$

45,1036

47,6 106 


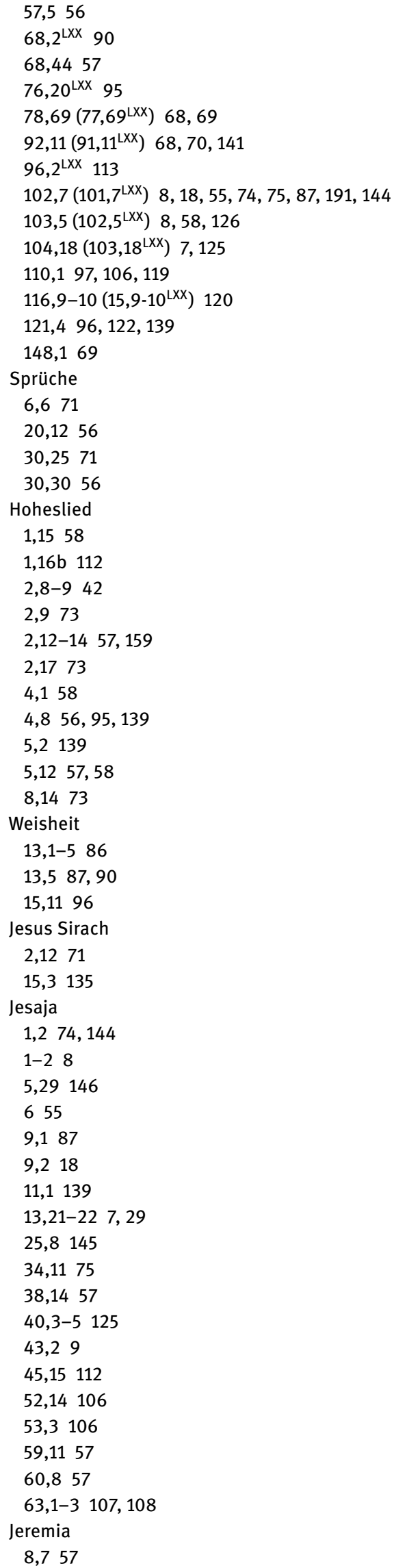

12,9155

17,11 151

23,24 121

48,28 57

Ezechiel

1,1095

23,2057

29,397

37,996

Daniel

39

5,2157

Hosea

$5,1456,146$

6,276

7,11 57

Amos

$1,256,146$

$3,856,146$

Jona

2,1 76

Micha

1,1658

Nahum

2,857

Zefanja

2,17 75

Sacharia

$6,129,11$

9,9-10 57

Maleachi

3,20 11

\section{Neues Testament}

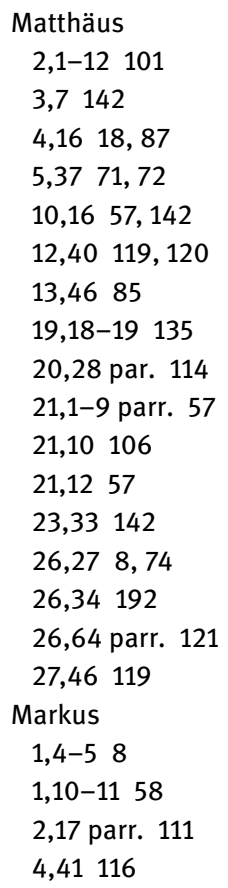


10,44118

11,15 parr. 57

14,36119

15,34119

16,19139

Lukas

$1,6968,70,97,141$

1,7811

$1,7918,145$

2,2457

3,38

11,37113

12,3018

$12,3218,87$

23,43.46 119-121

24,45 par. 91

Johannes

1,1 109, 119, 121

1,1-3 91

$1,1-18102,126,133$

1,597

1,8109

$1,1468,91,95,97,110,112,115-117,119,121,139,141,191$

1,2942

2,2185

3,13 117

3,14-15 142, 143

3,31 117

4135

4,10-15 134, 135

6135

7,37-38 134, 135

8,23117

8,469

8,59 96, 97, 101

10,17-18 119, 144

11,43146

12,2685

12,35-36 97, 101

15,1-8 29

16,1385

17,5121

19,33-34 119

19,34 122, 134, 135, 144

20,2296

20,28109

21,2583

Apostelgeschichte

2,33 121

7,55-56 122

13,248

19,98

27,12 144

27,4325

Römerbrief

1,20 12, 86, 110

$1,20^{\mathrm{Vg}} 111$

1,258

7,1485

8,3112
9,487

1. Korintherbrief

2,6-8 101, 106, 114

2,6-16 100, 101

2,8 96, 100, 101, 105, 114

2,9 98, 113

2,159

9,9-10 24

$9,2298,100,103,104,108,117,118$

13,29

$15,3-576$

15,2497

$15,4590,118$

15,47-48 145

15,55145

2. Korintherbrief

1,17-18 71, 72

3,685

$5,2118,87$

8,998

Galaterbrief

4,21-5,1 84

5,2236

Epheserbrief

1,21-22 95, 97

1,23102

$3,10108,113,114$

4,10117

4,22-24 11

6,12108

6,16135

19,1-3 101

Philipperbrief

2,6-8 90, 96, 98, 102, 103, 110, 112, 118

Kolosserbrief

1,15-20 95, 97, 102, 103, 139

2,15 114

2,9 110

$2,9^{\mathrm{Vg}} 111$

3,9 11

1. Thessalonicherbrief

5,23119

1. Timotheusbrief

2,4 90, 118

$3,16103,110,112,121$

2. Timotheusbrief

2,1942

Titusbrief

2,13 109

Hebräerbrief

1,8-9 109

2,14104

4,15 113

10,20113

Jakobusbrief

1,7-8 71

1. Petrusbrief

3,19 107

$3,2268,95,97$

4,6107 


\author{
5,8146 \\ 2. Petrusbrief \\ 1,1 109 \\ Offenbarung \\ 4,795 \\ $5,586,95,96,138,143,145,191$ \\ $5,5-6,1141,143$ \\ 5,696 \\ $5,12^{\mathrm{Vg}} 111$ \\ 9,19143 \\ $12,7-963$ \\ 12,14-15 143 \\ 12-13 143 \\ 14,6103 \\ 16143 \\ 20143 \\ 20,2143
}

\section{Physiologus}

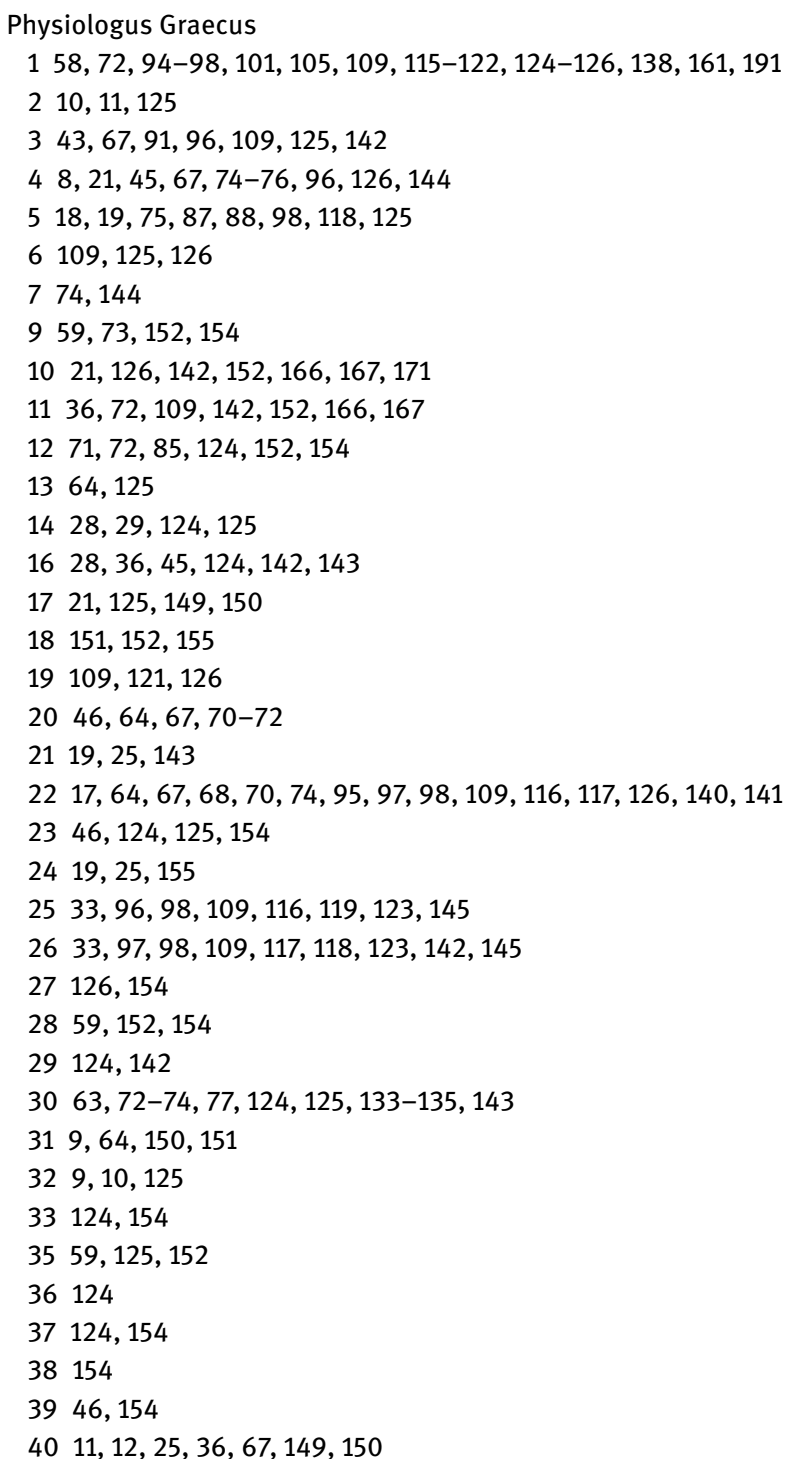

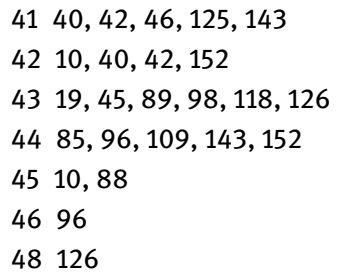

Physiologus Bernensis siehe Namen- und Sachregister

\section{Weitere Autoren und Schriften}

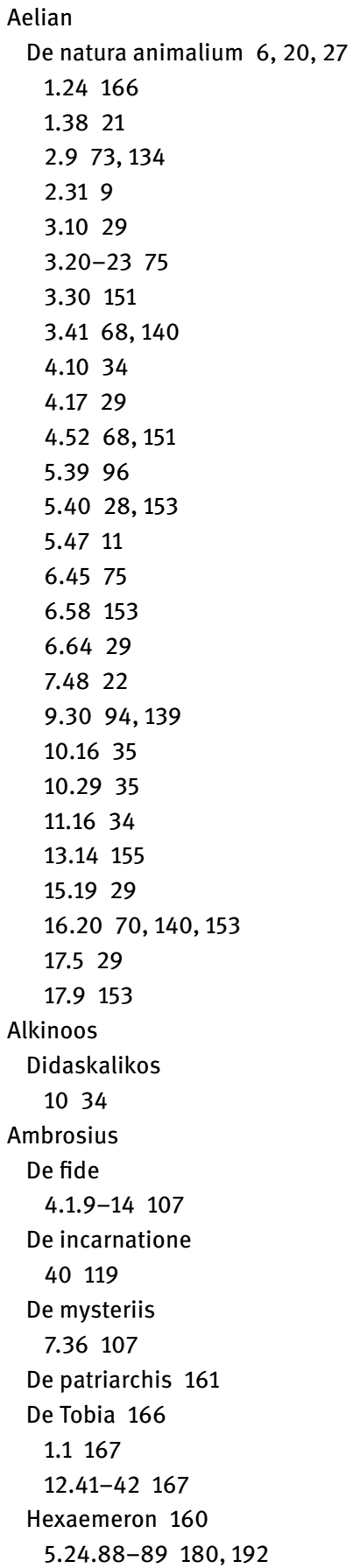


Anastasios Sinaites

Hexaemeron

4.5.5 113

12.5 .1113

Ps.-Anastasios

Adversus ludaeos dialogus alius 113

Adversus ludaeos disputationes

2113

Anaxandrides

frag. 4022

Andreas-Akten 149, 155

(A) Martyrium Andreae prius 16110 Anthologia Palatina
6.16929
6.4529

Apion

Aiүuптıкко́ 23

Apollonios von Tyana

Mirabilia

46155

Aristeasbrief 24

142-169 23

Aristophanes

Vögel 21

Aristoteles

De anima 30

2.2.413a26-414a3 30

2.4.415a26-b7 19

3.2.426a20 84

3.4.429a10-429b10 30

De arte poetica

$1.1447 \mathrm{~b} 196,84$

De generatione animalium 19, 29

2.1.731b24-35 19

3.6.756b13-757a13 19

5.6928

De incessu animalium 19, 29

9.709a9-10 19

De motu animalium 19, 29

De partibus animalium 6,19, 29

1.1.641a7 84

1.519

3.2.663a22 140

Historia animalium 6, 19, 29, 151

2.1.498a9 19

2.1.499b20 140

5.19.552b15 9

5.34166

6.4.562b 152

6.32.579b16-30 19

8(9).6.612a 153

8-9 21

9.6.612a12-15 28

9.34.619b18-23 18

9.44.629b35-36 95, 139

Metaphysica

1.5.986b14 84

1.8.990a3 84

Ps.-Aristoteles

De audibilibus 29
De mirabilibus auscultationibus 29

10152

De mundo

392a31-33 33

Artemidor

1.79124

3.16124

Ascensio Isaiae 101, 102, 106, 123

6-11 96, 100, 101

Asterios

Homilia in Psalmum

15.16109

Athanasios

De incarnatione

21.3-22.5 119

frag. bei Theodoret, Eranistes Florilegium

1.39112

2.25a 121

Oratio contra Arianos

1.61.1-62.1 121

2.8 .1112

2.13 .1121

3.50 .2112

3.67 .6112

Vita Antonii

9154

50-52 154

Ps.-Athanasios

De incarnatione contra Apollinarium

1.18120

2.3112

2.14120

2.15121

In occursum Domini

4121

Quaestiones aliae

20114

Athenaios

Deipnosophistae

3.101a 84

Atramchasis-Epos (altbabylonisch) 144

Augustin de Dacia

Rotulus pugillaris

1.256.19 91

Augustinus

Contra Faustum

32.20165

De civitate dei 164,165

7.1111

21.2-5 164

De doctrina christiana $162,164-166,168-171$

1-3 163

2.7.10 163

2.166

$2.16 .24162,166$

2.24.59 166

2.39.58-59 163

De genesi ad litteram

5.16 
Enarrationes in psalmos

$45.76,165$

Epistulae

43.25165

Sermo

337.1108

Barnabasbrief 24-26, 85

5.6109

5.10-11 109

10.1-3 86

10.5-11 25

10.7155

Basileios von Caesarea

De Spiritu Sancto

15121

Homiliae in Psalmos

14.1.3 166

Basileios von Seleukia

Orationes

23.1113

28.1113

Caesar

De bello Gallico

6.2789

Cassiodor

Institutiones divinarum et saecularium litterarum 170

Catena siehe Katenen

Celsus siehe Kelsos

Chaldäische Orakel

frag. 3795

Cicero

De natura deorum

2.123-7 30

Clemens siehe Klemens von Alexandrien

1. Clemensbrief 85

2. Clemensbrief

1,1 109

Collectio Sabbaitica (=Akakios, Epistulae)

8113

Decretum Gelasianum 160, 161, 166

6.11160

Didymos (?)

De trinitate 2.7.8.9 104

Diodor

Bibliotheca historica

1.821

Diogenes Laertios

Vitae philosophorum

1.1023

7.18784

Dion von Prusa

Orationes

12.3534

Ps.-Dionysios Areopagites

De caelesti hierarchia

15.895
Epikur

Sententiae (Gnomologium Vaticanum Epicureum)

2984

Epiphanios

Adversus haereses

21.2.499

Epistula apostolorum 102

11-14 100

Euseb

Commentarius in Isaiam

2.28112

Commentarius in Psalmos

23 106, 108

Demonstratio evangelica

5.4.4-9 112

Fredegar-Chronik 180, 183, 184

Gelasius

Historia ecclesiastica

2.19.26 113

Gellius

Noctes Atticae

5.1422

Ginza

Rechter Teil, 5.138-14699

Gregor der Große

Epistula ad Leandrum

1169

2169

Moralia in Job

$15.19170,171$

Gregor von Nazianz

Epistula

101.1697

Orationes

24.9113

32.18112

39.13113

40.10113

45.22113

45.25108

Gregor von Nyssa

Adversus Apollinarium 120

Contra Eunomium

3.3.43 121

Epistula

3.22120

Homilia 4 in Canticum canticorum GNO 6, 108112

Homilia 5 in Canticum canticorum 2.15108

Homilia 11 in Canticum canticorum

24109

In ascensionem Christi 108

324.11-12 108

326.2-6 108

In Christi resurrectionem

1 112, 114, 120 


$$
\begin{aligned}
& \text { Oratio catechetica } \\
& \begin{array}{l}
23.3-26.1112 \\
25.1108 \\
32.6108
\end{array}
\end{aligned}
$$

Hermas, Hirt des

$$
\text { 40.4-6 } 110
$$

Herodot

Historiae

$$
2.73153
$$$$
3.109166
$$

Hesych

In pascha homilia

$$
1.5107
$$$$
\text { 2.2-3 } 121
$$

Hieronymus

Adversus lovianum

1159

Commentariorum in Amos Prophetam

$$
\text { 7.7-9 } 10
$$

De viris illustribus

$$
722
$$

Epistula

124.12103

Sermo paschale

2109

Vita Pauli 154

8153

Hippolyt

frag. 20 in Ps 23,7 (= Theodoret, Eranistes Florilegium 2.16) 107

Refutatio omnium haeresium

$$
\text { 6.19.698 }
$$

Ps.-Hippolyt

Contra Beronem et Heliconem haereticos 111

De pascha

frag. 3120

De teophania

$$
4113
$$

Horapollon

Hieroglyphica

$$
1.1996
$$

Hugo von St. Victor

Eruditio didascalica

6.585

Ignatius

Epistula ad Ephesos

19114

19.2107

Ps.-Ignatius

Epistula ad Antiochenos (recensio longa)

5.1110

Irenaeus

Adversus haereses

1.23.3 98

1.26.1 119

1.30.4-699

2.30 .3106

3.8.3 106

3.16 .8106
4.33.13 106

Demonstratio apostolicae praedicationis

84-85 106, 107

Isidor

De ecclesiasticis officiis

1.45179

Etymologiae

12.1192

12.1.42-43 180

Joasaph von Ephesos

Epistula Joasaphi ad Nilum

501-548 113

Johannes Chrysostomos

De incomprehensibili dei natura

3.53-193 108

4.133-158 108

Homiliae in Matthaeum

36.11.1-2 166

Ps.-Johannes Chrysostomos

De ascensionem Domini

4108

De Pharisaeo 113

Homilia 5 in Psalmum 96113

In annuntiationem et contra Arianos 113

In assumptionem Domini 113

In centurionem 113

In sanctum pascha

6.61107

Oratio de nativitate

1113

Johannes von Damaskus

De fide orthodoxa

75122

Johannes-Akten 149-151, 154, 156

56-57 151

60-61 151

Johannesapokalypse

1111

13111

Justin der Märtyrer

Apologia

1.51.6-7 105

1.65 .1124

Dialogus cum Tryphone 85, 95

36.3-6 105

85.1-4 105

9197

Justinian

Anathemata synodis Constantinopolitani, Anathema 7 103, 104

Epistula ad Menam, Anathema 4 103, 104

Katenen

Catena in Epistolam ad Philippenses

г 113

Catena in Evangelium S. Lucae

$23.43 \sqcap 120$

Fragmenta in Joannem (in catenis)

146119 


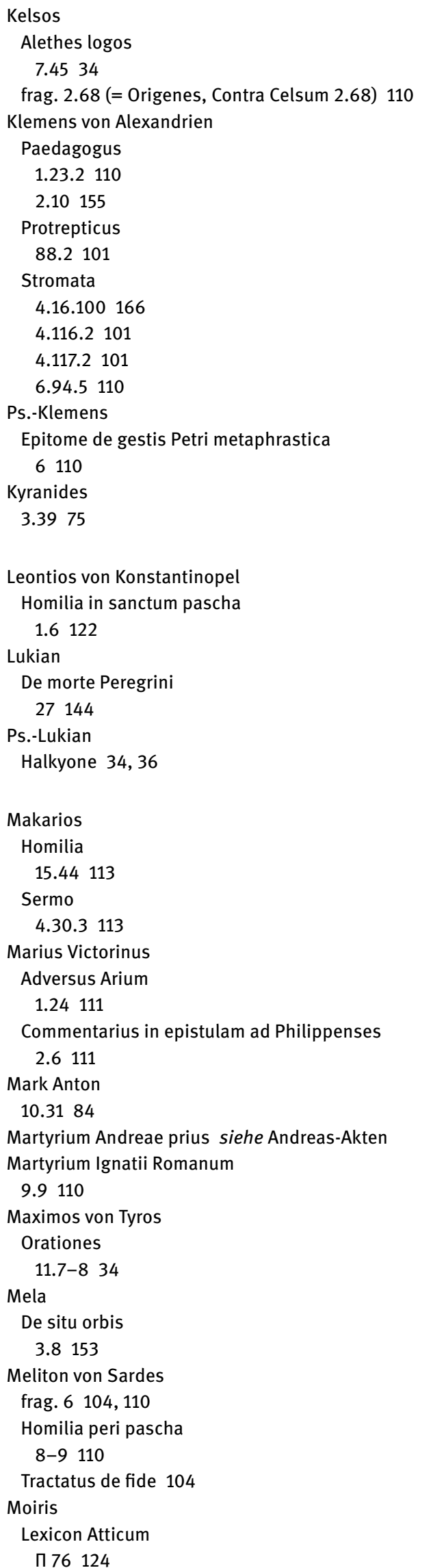

Mure, Konrad von

De natura animalium 184

Nag Hammadi Codices (NHC)

Der zweite Logos des großen Seth (NHC 7,2)

56.21-29 99

Die dreigestaltige Protennoia (NHC 13,1)

47-49 99

Eugnostosbrief (NHC 3,3/5,1)

$3 / 299$

Noēma (NHC 6,4)

41-42 99

Philippus-Evangelium (NHC 2,3)

57.28-58.3 99

67.9-12 100

Sophia Jesu Christi (NHC 3,4)

9499

Zostrianos (NHC 8,1)

13099

Neilos (?)

De vitiis

2124

Nikarchos

Epigrammata

AGr 11.16619

Nikodemusevangelium 145

21107

2563

Novatian

De trinitate

11.1111

11.4111

12.5111

Oden Salomos

42.3-4 101

Oppian

Kynegetika

2.236-240 73, 134

3.183-207 151, 152

Oracula Sibyllina

8.291-292 101

12.32101

Origenes

Commentariorum series in Matthaeum

27112

76114

92114

125114

Commentarius in epistulam ad Romanos (Rufin)

1.6.2 103

5.7.5 103

Commentarius in lohannem

1.107110

1.216-219 103

2.168-170 97

6.288107

19.38103

frag. 18112 
Commentarius in Matthaeum

12.18114

12.30114

16.8114

16.19 106, 107

28103

40114

De pascha

2.28-30 107

De principiis 103

3.3.2 114

Dialogus cum Heraclide

5-8 119

Excerpta in Psalmos 107

17112

Fragmenta in Lamentationes 107114

Homilia 2 in Psalmum 15107 105.19-21 107

Homiliae in Genesim

$\begin{array}{ll}8.8 & 103 \\ 9.3 & 114 \\ 14.1 & 103\end{array}$

14.1103

Homiliae in Isaiam

3.2107

Homiliae in Leviticum

1.1112

1.3103

Homiliae in librum ludicum

7.2107

Homiliae in Lucam

$$
\text { 6.4-6 } 114
$$

Selecta in Psalmos 114

Ovid

Metamorphoses

2.531-532 34

$11.410-748 \quad 35$

14.53834

15.392153

Pamphilos

Apologia (Rufin)

143107

Paulus-Akten 123, 149, 154, 156

Petrus-Akten 149, 154, 156

Petrusapokalypse

17105

Petrusevangelium

19119

Phaedrus

Fabulae

56322

Philippus-Akten 149

Philodemos von Gadara

De musica

108 Kempke 84

Philon von Alexandrien

De animalibus 21, 35

De Josepho

14533

\author{
De migratione Abrahami \\ 173-174 105 \\ De opificio mundi \\ 23105 \\ De somniis \\ 1.238105 \\ De specialibus legibus \\ 4.100-118 24
}

Quis rerum divinarum heres sit

$$
\text { 127-128 } 58
$$

Philon von Byblos

FGH 790F4 22

Philostrat

Vita Apollonii

3.49153

Phlegon von Tralleis

De rebus mirabilibus 34-35 153

Pistis Sophia

1.11-13 99

799

10-1499

Platon

Kratylos

414c 23

435c 23

Politeia

430b8 21

$488 \mathrm{c} 23$

$588 \mathrm{~b}-589 \mathrm{c} 154$

Protagoras 21

Plinius

Naturalis historia 6, 27, 159, 164

8.1321

8.2489

8.46152

8.5622

$8.6228,153$

8.71140

8.76140

8.88-89 97

$8.11873,134$

8.13328

8-11 20

10.2153

10.34159

10.62.169 166

10.67.188 9

10.89-91 35

10.11829

11.255140

29.4.76 9

29.129-131 11

37.15.55-61 10

37.57152

37.1907

Plutarch

De esu carnium 31

De Iside et Osiride

362B 23 
380F 23

381A 25

$382 \mathrm{AB} \quad 23,34$

74-76 22

De recta ratione audiendi

43A 23

De sollertia animalium 21

960A 31

966D-973A 29, 32-34

974C 28

975AB 34, 36

976BC 34

976D 28, 153

981F-983F 33, 35

$985 \mathrm{C} 31$

1821

Gryllus

986C-992C 31, 33

992E 34

Quaestiones convivales 670BC 96

Porphyrios

De abstinentia 21

$$
\text { 2.22-24 } 21
$$

Proklos

In Timaeum 3.158.6-7 95

Prokop

Commentarius in Isaiam 113

Prudentius

Hamartigenia

553-556, 569-573, 581-584 168

608-610, 613-614, 617-620 168

Quaestiones Bartholomaei

11-15 107

Rufin von Aquileia

De benedictionibus patriarcharum 161

$1.6125,161$

Sedrachapokalypse

12-16 111

Seneca

De beneficiis

2.19 .122

Dialogi

3.3.6 30

4.3.130

Epistulae

41.134

71.3230

Naturales quaestiones 27

Sextus Empiricus

Adversus mathematicos

732

Pyrrhonische Hypotyposen

1.835

1.2935

$1.62-7831,32$
Solinus

Collectanea rerum memorabilium 27.27152

Stobaios

Florilegium

1.3.9 122

Strabon

Geographica

17.2418

Synesios

Hymnus 6

24-32 121

Tabula Cebetis

23.1-2 154

Tatitus

Anales

6.28153

Terenz

Andria

61163

Tertullian

Adversus Praxean

30.5111

Apologeticum

5.2111

21.30-31 111

De anima

34.498

De carne Christi

14104

Scorpiace

9.1111

Thekla-Akten 12, 149-151, 156

40150

Theodoret

Commentarius in Psalmum

23.7-10 108

Curatio graecarum affectionum

6.77113

De incarnatione Domini

14113

18112

Eranistes

Dialog 1113

Oratio de providentia

10113

Theokrit

Eidyllion

7.21-22 11

Theon

Rhetorica progymnasmata

12124

Theophilos von Alexandria

Epistula paschalis

= Hieronymus, Epistula 96.10104

Epistula synodica

= Hieronymus, Epistula 92.4104

Theophrast

De historia et causis plantarum 6 


\section{De igne}

609

Thomas-Akten 149, 151, 152, 154-156

8151,156

50152

70152

79152

80111

91152

108-113 152

\section{Valentin}

frag. 3 (= Klemens von Alexandrien, Stromata 3.59.3) 110 Vinzenz von Beauvais

Speculum naturale

Prolog 13161

Vita Adae et Evae 123, 126

Vita sancti Symeonis 180,184

\section{Xenophon}

Kyropädie

2.321 
